\title{
SOME INEQUALITIES FROM SWITCHING THEORY ${ }^{1}$
}

\author{
BY J. L. BRENNER
}

Communicated by Irving Reiner, February 13, 1969

The following inequality was discovered by Turner and Conway (SIAM Rev. 10 (1968) 107).

Let $0<p<1,0<q<1, p+q=1, m>1, n>1$. Define $F(p, q)$ $=\left(1-p^{m}\right)^{n}+\left(1-q^{n}\right)^{m}-1$. Then $F(p, q)>0$. Their derivation is based on reliability theory. The following variants and generalizations are given. First, the inequality is reversed if $0<m, n<1$. Second, the two finer estimations

$$
\begin{gathered}
F(p, q) \geqq \sum_{r}\left(\begin{array}{l}
n \\
r
\end{array}\right) p^{m n-n-m r+2 r} q^{n+m r-2 r}, \\
{\left[1-p^{m}-\left(\begin{array}{c}
m \\
1
\end{array}\right) q p^{m-1}-\cdots-\left(\begin{array}{c}
m \\
r
\end{array}\right) q^{r} p^{m-r}\right]^{n}+\left[1-q^{n}\right]^{m}} \\
+\left(\begin{array}{c}
m \\
1
\end{array}\right) q^{n}\left(1-q^{n}\right)^{m-1}+\cdots+\left(\begin{array}{c}
m \\
r
\end{array}\right) q^{n r}\left(1-q^{n}\right)^{m-r}>1
\end{gathered}
$$

hold $(0<r<n)$. Next, let $p_{i}, m_{i}$ be a set of $k$ positive numbers; let $r$ be nonnegative and suppose $n \geqq 1$. Suppose further $\forall_{i}\left\{m_{i}>1\right\}$, $n \cdot \prod m_{i}=S, r+\sum p_{i}=1$. Then if $r=0, n=1$, we have

$$
\sum\left(1-p_{i}^{S / m_{i}}\right)^{m_{i}}>k-1 \text {. }
$$

If $r>0, n>1$ (and in certain other cases) we have

$$
\sum\left\{1-\left(1-p_{i}\right)^{S / m_{i}}\right\}^{m_{i}}>\left(1-r^{n}\right)^{S / n} \text {. }
$$

Contrary to the proof given by reliability theory, the combinatorial proofs of this paper are symmetric in the parameters. Independent analytic proofs involve induction, together with estimation of the minimum of a nonconvex function. This article will appear in Journal of Combinatorial Theory.

University of Arizona, Tucson, Arizona 85721

${ }^{1}$ Sponsored by National Science Foundation under grant GP-9483. 